\title{
PRODUCTION OF NEGATIVE PIONS AND THEIR INTERACTIONS IN TISSUE
}

\author{
V. P. BOND \\ Brookhaven National Laboralory, Upton, N.Y., U.S.A.
}

\section{MASTER}

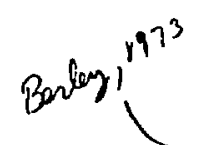

A number of aricles on the possible uses of negative pi mesons in radiotherapy are available (Bond, 1971; Boyd, 1971; Fowler and Perkins, 1961; Kaplan, 1969; Raju and Richman, 1972). The intense beams of negative pi mesons required for radiotherapeutic application are produced by causing either encrgetic protons or electrons to impinge on a target It is possible to use a wide variety of target materials; beryllium or a higher $\mathbf{Z}$ metal have been employed. Although pions are produced at lesser encrgies, usually particles of 500 or more MeV are used in order to obtain the intense pion beams required. The crosssection for pion production with an electron beam is of the order of 30 times less than for a proton beam; however, this varies with projectile energy, pion energy, pion angle, target material, etc. Greater pion yields can be obtained with higher energy protons if thicker targets are used. Electrons with the same momentum as the pions are produced in the target and are initially present in the beam. These can be removed almost quantitatively by clectromagnetic means, before the beam impinges on tissue.

The negative pi meson has a mass 273 times that of the electron, or approximately $15 \%$ of that of the proton. Pions are unstable particles with a charge of minus one that decay with a mean life of $2.6 \times 107$ seconds (rest frame), to form a muon and a neutrino. Practically, some 5 to 10 meters minimal distance from the target to the experimental arca is required because of the need for shielding and for beam optics equipment. There is a considerable loss of pions over this distance due to decay as well as capture and scattering of the pions if shiclding or other absorber is introduced into the beam. Muons and clectrons are produced, which are retained as contamination of the beam if their momentum is equal to that of the pions. Muon contamination should be a minor problem; electrons can be removed either by clectromagnetic means or by the use of absorbers.

In tissue, pions give up their kinetic energy in interactions with atomic electrons. Thus along most of the path of a pi meson in matter, they behave somewhat like an eleciron as regards rate of energy loss, or $\mathrm{dE} / \mathrm{dx}$ (however, the values of $\mathrm{dE} / \mathrm{dx}$ for electrons and pions of the same energy are different). Because its mass is intermediate, it scatters less than does the electron but more than the proton. The degrce of scattering depends on energy. Near the end of its range the slowed down meson is captured in atomic electron orbits to forin a 'pi mesic atom', which then disintegrates. Neat the end of the particle track, the pi meson undergoes reactions with the nuclei of atoms, principally with carbon and oxygen. Of the remaining mass of $140 \mathrm{MeV}$, approximately $96 \mathrm{MeV}$ is converted 10 energy of emitted particles, and approximately $44 \mathrm{MeV}$ is used in overcoming the binding energy of the nucleus.

The cross-section for pion capture increases linearly with $\mathbf{Z}$. In soft tissue, about $73 \%$ of the pions are captured in oxygen, $20 \%$ in carbon, $3 \%$ in nitrogen and $4 \%_{\tau}$ in heavier ions. Results of measurements (Raju and Richman, 1972) to determine the types of particles produced 
TABLE I

Parricles produced ar the cond of piminus tracks in water

\begin{tabular}{lcc} 
Particle & $\begin{array}{c}\text { Average cnergy per } \\
\text { pion star(MeV) }\end{array}$ & $\begin{array}{c}\text { Averuge no. of } \\
\text { particles per pion star }\end{array}$ \\
\hline Prolons & 15 & 0.95 \\
Alpha particles & 6 & 0.99 \\
Ncutrons & 69 & 2.7 \\
Recril nuclei & 4.4 & 0.8
\end{tabular}

by pion-nuclear interactions in water, and the energy carried off by each type of particle, are shown in Table $\mathrm{I}$. For a beam of negative pions with an energy of $96 \mathrm{MeV}$. a momentum of $190 \mathrm{MeV} / \mathrm{c}$, the range in tissue is approximately $25.5 \mathrm{~cm}$.

The negative pi meson beam thus, by undergoing $\mathrm{dE} / \mathrm{dx}$ losses much as beams of other particles, produces a Bragg curve in tissue or other matter. In addition, because of nuclear disintegrations confined largely to the end of the range of the negative pi meson, additional energy in the form of high LET radiation is liberated in the region of the peak of the Bragg curve] of the ncgative pi mesons. Such a primary Bragg peak of the negative pi mesons, and the added energy from star formation at the end of the range is shown in Figure ! (Bond, 1971). The curve is for a pure negative pi meson beam with an energy of $96 \mathrm{MeV}$, a momentum of $190 \mathrm{MeV} / \mathrm{c}$, and a momentum sprcad of $5 \%$. The peak to plateau ratio is approximately 3 to 1 , and the full width at half ma ximum is ahour $4 \mathrm{~cm}$.

Because the plateau region of the depth-dose curve results mainly frnm $\mathrm{dE}: \mathrm{dx}$ losscs of the negative pions, the LET is low, similar to that of cnergetic electron beams. In the peak region, however, because of the liberation of charged particles and neutrons, the LET is higher. The range of LETs is quite broad, extending from a fraction of $1 \mathrm{keV}_{!} \mu$ due to clectrons and muons, to values approaching $1000 \mathrm{kcV} ; \mu$ due to recoil nuclci such as carbon.

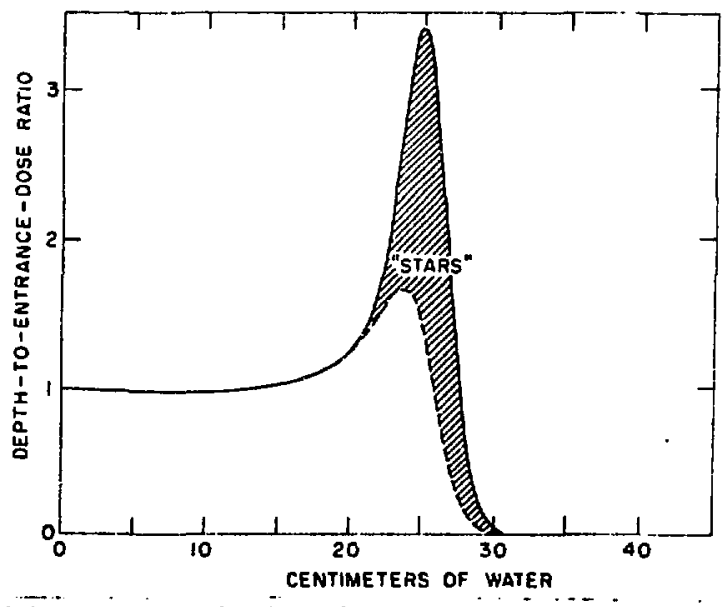

Fig. 1. Depith-dose curve in tissue for a hram of pure pi minus mesons. Momentum ign Mevic $=5 \%$.

(Calculated by S. Curtis. personal communication.) 
- The mean LET has been shown to be comparable to that of fasi neutron heams with wiean energies of several MeV.

The peak region of the depth-dose curve shnwn in Figure 1 is too narrow for most purposes in radiotherapy. Thus usually the peak tvould be widened by increasing the momcrtum spread of the beam. In doing so. however, the pcak in platcau ratio of the depth-dase curve is reduced. However, with the peak region covering a $10 \mathrm{~cm}$ span. the peak to plateau ratio is still of the erder of 1.5 or 2,101 . Because the high I.ET region of the beam has a higher RBE than does the plateau region, the ratio of 'effective dose' (dnfe $\times$ RBE) in the peak to plateau region is of the order of 2 to 3 times these values.

With the use of a proton beam to generate pions, tlie beams of pions can be very intense and yield cose rates of the order of 80 rads/min due to pion stars over a tumor volume of $1000 \mathrm{~cm}^{3}$. With this intensity and dose rate a single pion beam, directed horizontally. vertically, or either as desired, can be used for radiotherapy.

If electrons are used to generate pions, then because of the relatively small cross-section for pion production, 2 much larger solid angle of pion beam must be accepted in order to obtain the necessary intensity and dose rate. This approach is being investigated at the Stanford facility, in which a beam of $500 \mathrm{MeV}$ electrons with a cursent of one milliampere is allowed to impinge on a metallic target. Pions cmitted over a $360^{\circ}$ sector are then entrained into 60 separate beams emerging at roughly a $90^{\circ}$ angle to the electron beam path. These beams are then bent electromagnetically to form a large 'cylinder' of beams, which are then again bent through $90^{\circ}$ to allow them to ester, potentially from the full $360^{\circ}$ expanse, normal to the surface of the patient placed horizontally partially within the 'cylinder' of beams. Exposure of a tumor volume is achieved by horizontal translation of the patient. Each beam must be controlied separaiely with respect to momentum and momentum spread.

The above properties of a pi meson beam lead to the twe presumed advantages of such beams for radiotherapy. First is that one can obtain good dose distributions, hoth laterally and in depth. This results in maximum energy deposition in the target tissucs with minimal energy deposition in normal tissucs. Also, the fact that the beam has a well defined range allows one to stop the heam just short of vital structures. $A$ second advantage is the

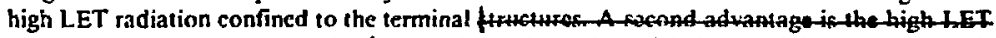
fadiation parts of the charged particle beam. This leads to a high RDE with a correspondingly greater peak to plateau ratio of 'cffective dose' (dose $\times$ RBE), and a low OER that has the potential for partially overcoming the problem cncountcred with conventional radiations, namely hypoxic but viable cells that are belicved in he present in many tumors and which are resistant to killing hy low-LET radiations.

As the negative pi meson cascades down the atomic elcctron levcls to be captured by the nucleus, characteristic $X$-rays are emitted. These are termed $\pi$-mesic $X$-rays. Most energetic and most easily detected are the $160 \mathrm{keV} X$-rays emitted when the negative pion interacts with an oxygen nucleus. Efforts have bren made, by detecting cither $\pi$-mesic $\mathrm{X}$-rays or gamina rays produced in the course of pron capture. 10 lacate the stopping region of the pion beam in tissue-equivalent materia:

No pion generators are as yet available for radiotherapeutic iriais. Beams to date have been of low intensity, and frequently contaminaied with elccirons. They have been suitable for dosimetry. A number of radiobiological experiments have been done; however, the low dose rates available have created problems in obtaining quantitative evaluations.

Several large pion facilities are being prepared for radiohiblogical and radiotherapeutic evaluations. These include the Los Alamos mieson production facility (1.AMPF), the Stanford University pjon generator, the Tri-univs:rsity meson facility (TRJUMF) machine in Vancouver, Canada and a facility in Zurich. Switzerland.

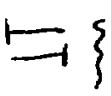


$+$

wither. $-\cdots$

plecese mention

P. tis at or $\rightarrow$ BERLEY. D. el ai. (1973): I.E.E.E. Trons. nucl. Sci., 20, 997.

delete

BOND, V. P. (1971): Amer. J. Roenigenol., III. 9.

BOYD. D. (1971): Stanfard Univ. Rep. HEPL 657.

FOWI.ER, P. H. and PERKINS, D. H. (1961): Nanure (Lond.). 189. 524.

KAPl_AN, H. S. (1969): In: Proceedings, III LAMPF U'sers Meering. 1969. LA 4397-MS,

Los Alamos Scientific Laboralory, Los Alamos, N.Mex.

RAJU, M. R. and RICHMAN, C. (1972): Curr. Top. Radiat. Res., 8, 159. 